\title{
Successful Operation Following Neoadjuvant Immunotherapy Combined with Chemotherapy for Right Aortic Arch with Esophageal Cancer: A Case Report
}

\section{Zhilin Luo}

The third Affiliated Hospital of Chongqing Medical University

Tianhu Wang (D650221@hospital.cqmu.edu.cn)

Chongqing Medical University https://orcid.org/0000-0003-1494-3416

\section{Dong Li}

The third Affiliated Hospital of Chongqing Medical University

\section{Case report}

Keywords: Right aortic arch, Esophageal cancer, Neoadjuvant immunotherapy

Posted Date: April 19th, 2021

DOl: https://doi.org/10.21203/rs.3.rs-421468/v1

License: (c) (1) This work is licensed under a Creative Commons Attribution 4.0 International License.

Read Full License 


\section{Abstract}

Background Right aortic arch (AAA) with esophageal cancer is a kind of rare case. Tumor shrinkage after neoadjuvant immunotherapy combined with chemotherapy and subsequently successful excision of the esophageal tumor under minimally invasive surgery are reported for this case in this paper.

Case presentation The patient was a 50-year-old man who was diagnosed with AAA and esophageal cancer with a clinical stage of C-T3N1M0, stage III. Because Computed tomography(CT) indicated a larger volume of the tumor with signs of outward invasion, along with vascular malformations, neoadjuvant immunotherapy combined with neoadjuvant chemotherapy was given. After 3 cycles of neoadjuvant therapy, this patient achieved a fully clinical symptom remission, and re-examination result of chest CT showed tumor shrinkage, so a minimally invasive surgery was provided to remove the tumor. Following the surgery, immunotherapy and chemotherapy were continued.

Conclusion For locally advanced esophageal cancer with vascular malformations, neoadjuvant therapy is a good treatment option. If achieving downstaging, the tumor would be well removed just with a minimally invasive surgery.

\section{Introduction}

Immunotherapy, as an emerging therapy, has been applied to multiple tumors including esophageal tumor, and has shown good efficacy ${ }^{[1]}$. Here is a report that a patient, with RAA and esophageal cancer, was successfully operated following neoadjuvant therapy of Sintilimab combined with nanoparticle albumin-bound paclitaxel (nab-paclitaxel). The data are collected as follows.

\section{Case Presentation}

The patient was a 50-year-old man who visited our hospital on February 10, 2020 due to "progressive dysphagia for 3 months". Gastroscopy suggested a mass at $23-28 \mathrm{~cm}$ from the incisors, involving the full circumference of the esophagus. The biopsy provided a result of squamous cell carcinoma.

Endoscopic ultrasonography revealed involvement to the esophageal fibrous membrane. The result of a CT examination showed a RAA with a tumor in the middle and upper thoracic segments of the esophagus surrounded by enlarged lymph nodes (Fig. 1). Gastroscopic biopsy specimen were taken for Programmed Death Receptor Ligand-1(PD-L1) detection and the result provided a TPS of $10 \%$. Cranial Magnetic Resonance Imaging(MRI), bone scan, and abdominal CT showed no sign of metastasis, with the clinical stage as $c-T_{3} N_{1} M_{0}$, stage $\Downarrow$. Because $C T$ indicated a larger volume of the tumor, with signs of outward invasion along with vascular malformations (Fig. 2), neoadjuvant immunotherapy combined with neoadjuvant chemotherapy was given considering the great difficulty to perform a surgery directly. The treatment regimen is described with details below: 1 . Sintilimab $200 \mathrm{mg}$, once every three weeks ${ }^{[2]} ; 2$. Nab-paclitaxel $400 \mathrm{mg}$ + Cisplatin $100 \mathrm{mg}$, once every three weeks $\left(1.6 \mathrm{~m}^{2}\right.$ for body surface area, 90 points for KPS score) ${ }^{[3]}$.After three cycles of neoadjuvant therapy, this patient achieved a fully clinical 
symptom remission and had been able to take the dry and hard food. Re-examination of chest CT revealed tumor shrinkage (Fig. 3). No distant metastasis was observed on the Positron emission tomography/Computed tomography (PET/CT). Therefore, after preoperative discussion, the surgical treatment was determined. There was no abnormal observation in preoperative cardiopulmonary function and other measures, without any surgical contraindications. Whereupon, on April 26, 2020, the patient underwent thoracic laparoscopy via left thoracic approach combined with esophagectomy + stomach replacing esophagus cervical anastomosis + lymph node dissection under general anesthesia. Findings during the operation include: superior mediastinal vascular malformation, left subclavian artery arising from the left to the right from the aortic arch, crossing above the esophagus (Fig. 4). The mediastinal pleura was opened along the periphery of the tumor to separate the space between the esophagus and the descending aorta, left subclavian artery, trachea, and left innominate vein; after freeing along the posterior wall of esophagus to the level of the left subclavian artery, it was difficult to continue on the esophageal tumor. Therefore, after dissection of paraesophageal lymph nodes, subcarinal lymph nodes, and left recurrent laryngeal nerve chain lymph nodes, the body position was changed, and an incision along anterior border of left sternocleidomastoid muscle was made at the neck by opening layer by layer, and fully freeing was made along the esophageal space until to the level of the left subclavian artery to completely removal esophageal tumor. Eostoperative pathology showed the involvement of esophageal tumor to the superficial muscular layer of esophagus and no metastasis observed in lymph node, with the postoperative stage as yp- $T_{2} N_{0} M_{0}$, stage I. The patient was discharged on the 8th day after the operation, without complications. Following the treatment of surgery, immunotherapy and chemotherapy were continued with the preoperative medication regimen. Up to the present, it has been 11 months after operation, and on the follow-up, the patient has no sign of recurrence or metastasis.

\section{Discussion And Conclusions}

RAA is one rare kind of macrovascular abnormality that may show no clinical symptoms and is often found by physical examination or chest CT at the medical visit due to other diseases, and some other patients may present with dysphagia, chest tightness, and dull pain ${ }^{[4]}$. When the RAA complicated with esophageal cancer occurs, it would be very difficult to perform the surgery directly because of the vascular variation. And the optimal surgical approach is the left thoracic approach. In this case, the mode

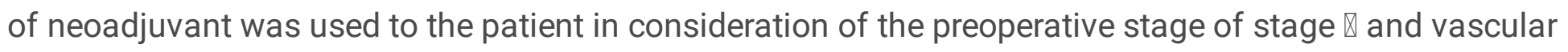
variation. With the rise of immunotherapy in recent years, it also shows remarkable efficacy in esophageal cancer ${ }^{[1]}$, so we chose chemotherapy combined with immunotherapy before the surgery. Reexamination of PET/CT, performed after the success of downstaging by neoadjuvant therapy, did not show metabolic uptake in mediastinal lymph nodes, so after the removal of esophageal tumor via left thoracotomy, beyond the routine dissection of paraesophageal lymph node, subcarinal lymph node and left recurrent laryngeal nerve chain lymph node, the surgical approach was not switched and the wound was not enlarged to perform the addtional lymph node dissection of the right recurrent laryngeal nerve chain. With this case, the author may reach the following conclusion: for patients with RAA and esophageal cancer, neoadjuvant chemotherapy combined with neoadjuvant immunotherapy is a good 
choice of treatment before surgery. If achieving downstaging, and no mediastinal lymph node metastasis on PET/CT re-examined, the same effect of conventional radical operation on esophageal cancer can also be achieved with the left thoracic approach. For skilled thoracic surgeons, the thoracoscope is enough to support the whole operation, but when three-dimensional reconstruction of the tumor and surrounding vessels can be performed before surgery, it would be more helpful for the operation and can allow surgeons to obtain a more visual understand on the course of variant vessels.

In conclusion, For locally advanced esophageal cancer with vascular malformations, neoadjuvant therapy is a good treatment option. If achieving downstaging, the tumor would be well removed just with a minimally invasive surgery.

\section{Abbreviations}

\section{RAA}

Right aortic arch

CT

Computed tomography

PD-L1

Programmed Death Receptor Ligand-1

MRI

Magnetic Resonance Imaging

Positron emission tomography/Computed tomography

$\mathrm{PET} / \mathrm{CT}$

\section{Declarations}

\section{Ethics approval and consent to participate}

This is a case report. The Institutional Review Board at The Third Affiliated Hospital of Chongqing Medical University has confirmed that no ethical approval is required.

\section{Consent for publication}

We obtained the patient's consent for publication of this case report.

\section{Acknowledgments}

We thank the patient for giving consent to this case report.

\section{Availability of the data and materials}

All the data are available in the patient's medical record. 


\section{Funding}

Not applicable.

\section{Author information}

\section{Affiliations}

Department of Thoracic Surgery, The Third Affiliated Hospital of Chongqing Medical University, Chongqing China, 401120.

Zhilin Luo,Tianhu Wang, Dong Li

\section{Contributions}

Tianhu Wang was responsible for this patient's operation, Zhilin Luo was responsible for conducted a literature search and drafted with the manuscript. Dong Li was involved in the management of the patient.

\section{Corresponding author}

Correspondence to Tianhu Wang.

\section{Competing interests}

The authors have no conflicts of interest relevant to this article.

\section{References}

1. Takashi Kojima MA, Shah,Kei. Muro,et al.Randomized Phase III KEYNOTE-181 Study of Pembrolizumab Versus Chemotherapy in Advanced Esophageal Cancer[J].Journal of clinical oncology: official journal of the American Society of Clinical Oncology,2020,38(35):4138-4148.

2. Wang HYang,KunlunWang,Tao,et al.The Combination Options and Predictive Biomarkers of PD1/PD-L1 Inhibitors in Esophageal Cancer[J].Frontiers in Oncology,2020,10: 300.

3. Wang YShi,RuiQin,Zhi-Kuan,et al. Nanoparticle albumin-bound paclitaxel combined with cisplatin as the first-line treatment for metastatic esophageal squamous cell carcinoma[J]. Onco Targets Therapy. 2013;6:585-91.

4. Sarv Priya,Richard Thomas,Prashant Nagpal,et al.Congenital anomalies of the aortic arch[J].Cardiovascular Diagnosis Therapy,2018 Apr; 8(Suppl 1): S26-S44.

\section{Figures}



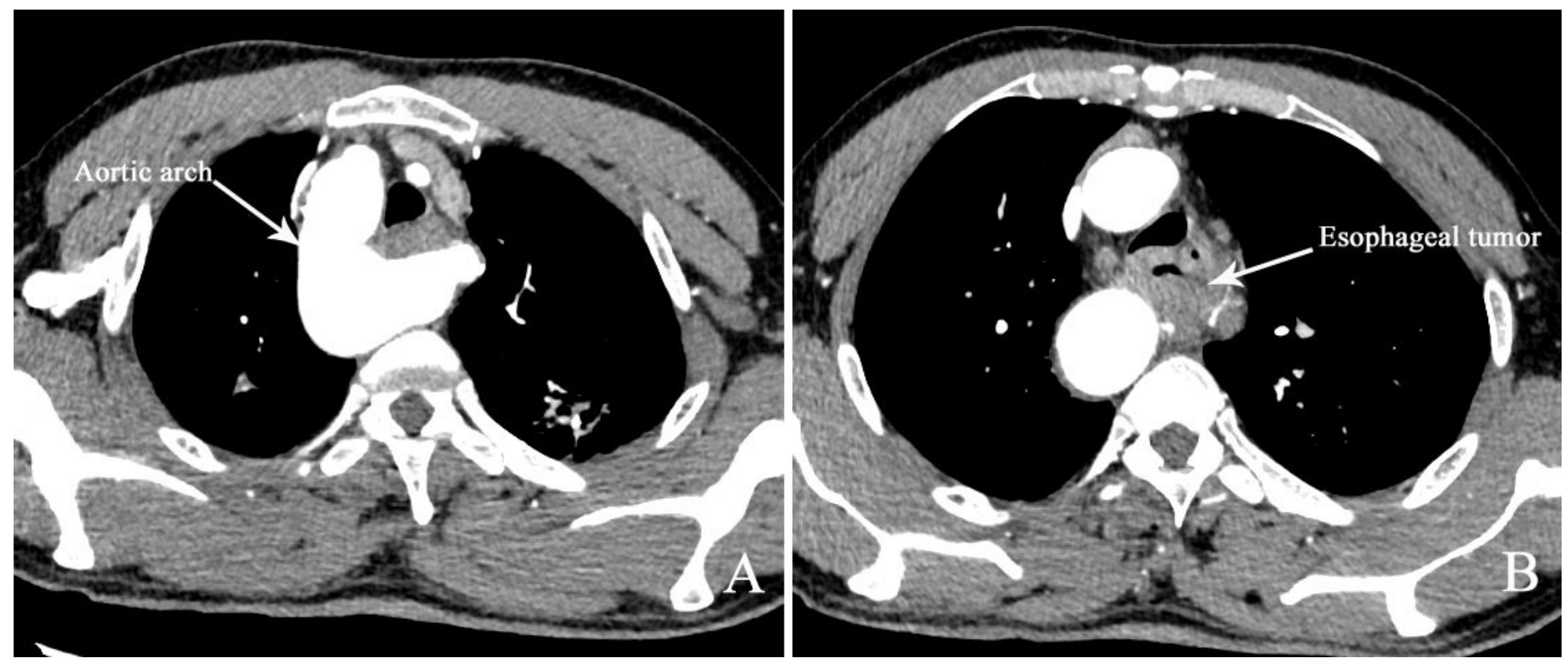

Figure 1

Right aortic arch (A) and esophageal tumor (B).
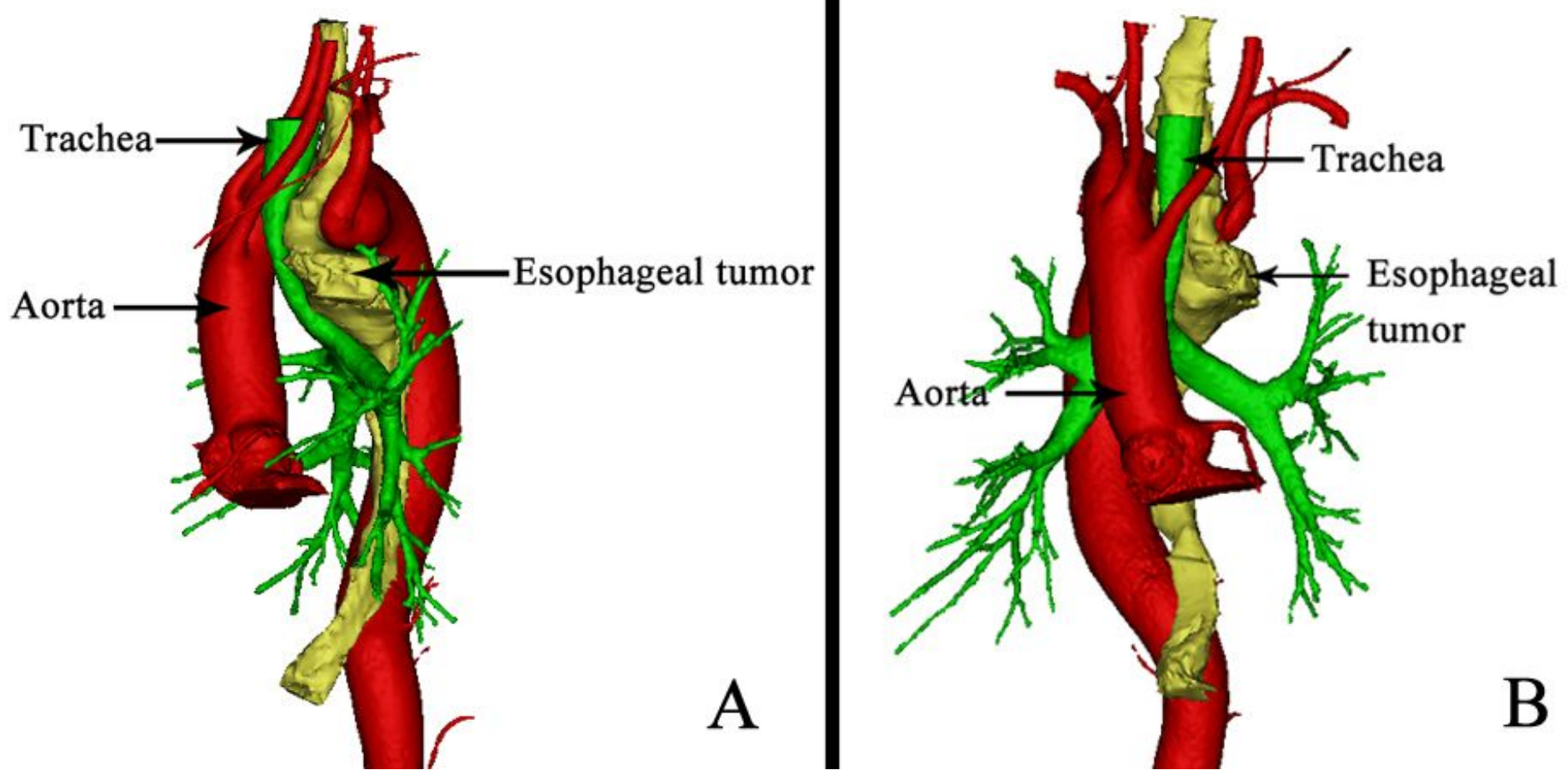

Figure 2

Three-dimensional reconstruction of esophageal tumor and surrounding tissue: lateral (A) and frontal (B) views. 

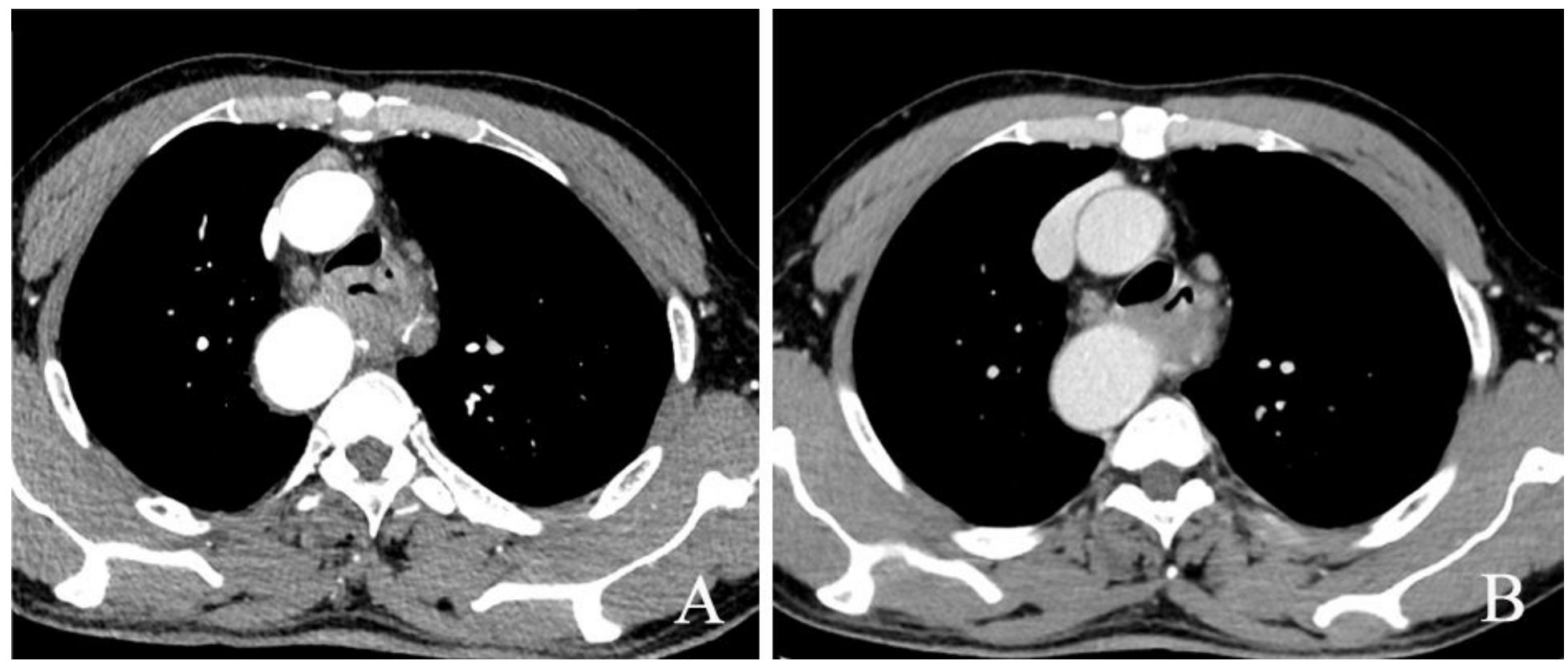

Figure 3

Before (A) and after (B) neoadjuvant therapy.

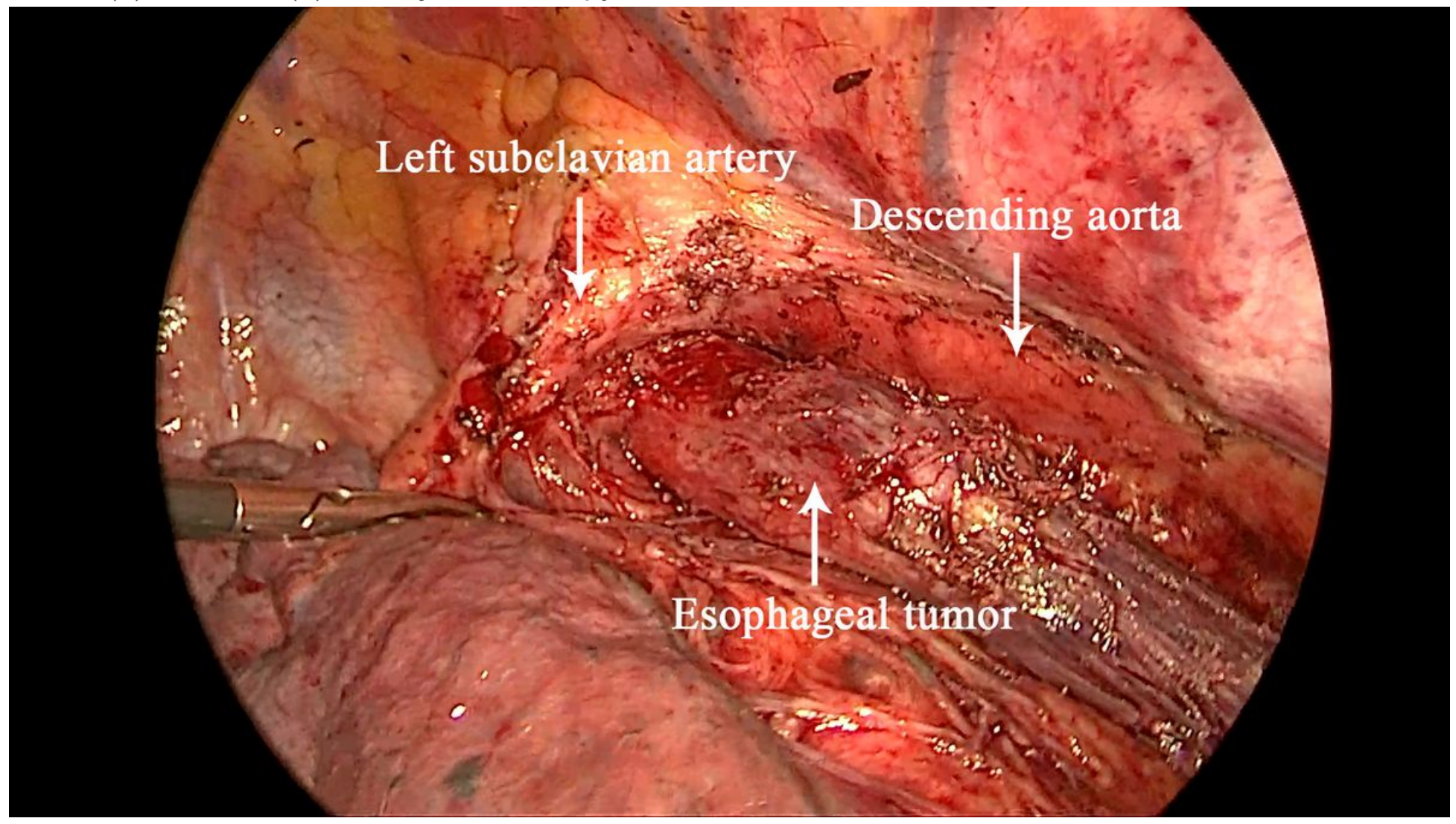

Figure 4

Intraoperative picture. 